\title{
A Nonlinear-Dynamics Based Approach to Stability Analysis of Non- Foster Networks with Electrically-Small Antennas
}

\author{
John F. McCann ${ }^{1,2}$, James T. Aberle ${ }^{2}$, Ying-Cheng Lai ${ }^{2}$ \\ ${ }^{1}$ Air Force Research Laboratory, Wright-Patterson AFB, OH, 45433, United States \\ ${ }^{2}$ Arizona State University, Tempe, AZ, 85287, United States
}

\begin{abstract}
Non-Foster networks have the potential to provide wideband matching to electrically-small antennas. Impedance data from a model of an electrically-small monopole provides the starting point of this work. A lumped-element model of the monopole is developed, and used to design a matching network using a shunt negative capacitor and $L$-section subnetwork. The resulting network provides a larger bandwidth than that with passive matching. Stability of the network is analyzed under the assumption of ideal negative capacitor, and it is demonstrated that a change in an antenna impedance can induce instability. Transient analysis using Agilent ADS software, calculations based on differential equations using MATLAB, and $s$-domain analysis are all employed. The network is similarly analyzed when the negative capacitor is replaced by an equivalent $J$-transform circuit section, allowing for the inclusion of a nonlinear negative resistance. Nonlinear differential equations are developed for general stability analysis of non-Foster networks.
\end{abstract}

Index Terms - Antenna matching, antennas, nonlinear microwave circuits, stability.

\section{INTRODUCTION}

The term non-Foster can be explained by referring to Foster's reactance theorem [1], which reads, in part, "The most general driving-point impedance obtainable by means of a finite resistanceless network is a pure reactance which is an odd rational function of frequency and which is completely determined, except for a constant factor, by assigning the resonant and anti-resonant frequencies, subject to the condition that they alternate and include both zero and infinity. Any such impedance may be physically constructed by combining, in parallel, resonant circuits, or by combining, in series, anti-resonant circuits." In fact, the impedance of any combination of ideal inductors and capacitors is a pure reactance with a positive slope versus frequency. When loss is included (resistors added to the circuit), the reactance function may have portions where the slope is negative. In these cases the real part of the impedance function is positive and the impedance rotates clockwise when plotted on the Smith chart. A non-Foster element or network is one that violates Foster's reactance theorem, and can be achieved using active components such as amplifiers or negative impedance converters. Non-Foster elements may be useful in matching to electrically-small antennas, in that the reactance of a nonFoster element can effectively cancel the reactance of an electrically-small antenna over a larger frequency range than other passive or active matching schemes.

In the past decade, there has been a large body of published work focused on non-Foster networks, particularly in the area of matching to electrically-small antennas [2, 3]. In [3], a model for an electrically-small monopole was used to design a wideband non-Foster matching network. The studies in [4] and [5] examined the design and analysis of non-Foster networks.

A main concern with non-Foster elements is stability. Many stability-analysis methods exist, some using equivalent circuits for negative impedance elements. The loss of information resulting from the use of equivalent circuits in non-Foster networks may lead to the failure to detect instabilities. Not only must a stability-analysis method be rigorous, but any equivalent circuits must contain sufficient information to allow the method to succeed in detecting phenomena such as hidden modes. Error may result from improper use of stability criteria $[3,6]$.

Small-signal techniques such as nodal or mesh loop analysis in the $s$-domain can identify the parameter values leading to poles in the right half-plane, resulting in exponential growth of current and voltage. However, non-Foster elements are generally nonlinear. The main idea behind this research is that nonlinear dynamics can be useful in more thoroughly analyzing the stability of non-Foster networks. The specific tasks are to identify a set of nonlinear differential equations that govern the behavior of the network and to determine the phase-space and parameter space to address the issues of stability, oscillations, and even chaos [7, 8].

\section{ANTENNA MODEL}

The input impedance of an electrically-small antenna generally consists of a low resistance and a large reactance. In the case of a dipole or monopole antenna, the reactance is capacitive. In our study, the antenna impedance data is the same as that used in [3], which represents the impedance of a $0.6 \mathrm{~m}$ monopole above an infinite ground plane for the frequency range $30 \mathrm{MHz}$ to $90 \mathrm{MHz}$. This antenna can be considered electrically-small, as it is 0.12 wavelengths at the center frequency of $60 \mathrm{MHz}$. Based on [9], a model for the monopole antenna was developed using a capacitor in series with a parallel combination of a resistor and an inductor. The 
calculated element values for the equivalent antenna model are $L_{A}=200 \mathrm{nH}, R_{A}=955.8 \mathrm{Ohms}$, and $C_{A}=8.54 \mathrm{pF}$. Figure 1 shows the comparison between the lumped element model as calculated in MATLAB.

\section{MATCHING NETWORK}

Because of the characteristics of the impedance of the antenna model, a passive network matching the antenna model to $50 \mathrm{Ohms}$ will be rather narrow band. A common method for passive matching is the L-section, described in Chapter 5 of [10] and used in [3]. Using such a matching network with two positive inductors, as shown in [3], centered at $60 \mathrm{MHz}$ and with $\mathrm{Z} 0=50 \mathrm{Ohms}$, the best match results in a bandwidth of $1.27 \%$, where bandwidth is defined as having reflection coefficient less than $-10 \mathrm{~dB}$.

If negative impedance elements are employed, it is possible to achieve a significantly larger bandwidth. In [3], a negative inductor and a negative capacitor are used in series (implemented using a floating negative impedance converter circuit) with the antenna to achieve a match across the entire range of $30 \mathrm{MHz}$ to $90 \mathrm{MHz}$. Here, only a shunt (and grounded) negative capacitor is used. This simplifies the analysis, and has the benefit that it is easier to ensure stability.

One goal is to design a network with the largest bandwidth possible. The design parameters are the negative capacitance value and the matching network elements. A MATLAB program loops through negative capacitance values, from $C_{N}=0 \mathrm{pF}$ to $C_{N}=30 \mathrm{pF}$. For each $C_{N}$ value, matching network element values are calculated based on the equations in [10], where there can be as many as four different matching networks possible for each load. For each matching network at each load (based on $C_{N}$ value), the percent bandwidth centered at $60 \mathrm{MHz}$ is calculated. The calculated bandwidth versus $C_{N}$ values for each type of matching network is shown in Figure 2. The maximum bandwidth possible is $5.75 \%$, with a negative capacitance of $C_{N}=15.86 \mathrm{pF}$. Note in Figure 2 that for a purely passive matching network $\left(C_{N}=0\right)$, the bandwidth is $1.27 \%$. Also note that for negative capacitance values from approximately $C_{N}=7.5 \mathrm{pF}$ to $C_{N}=15 \mathrm{pF}$, only matching networks of one type are possible. The maximum bandwidth found using this method is much larger than a passive matching network alone.

\section{NeTWORK WITH IdEAL NEGATIVE CAPACITOR}

The resulting non-Foster network is shown in Figure 3, which includes a lumped-element antenna model, shunt negative capacitance, and passive matching network. The lumped element values are listed in Table 1. Differential equations are developed using two node voltages, $v_{M}$ and $v_{A}$, and two branch currents, $i_{L M}$ and $i_{L A}$, as dynamic variables. The four differential equations that govern the behavior of the network are listed in Table 2 . These equations are solved in MATLAB using a simple time-step algorithm in increments of $0.1 \mathrm{~ns}$ for a $200 \mathrm{~ns}$ span. An initial condition of a $100 \mathrm{~mA}$ current through the antenna model inductor $L_{M}$ is applied. The
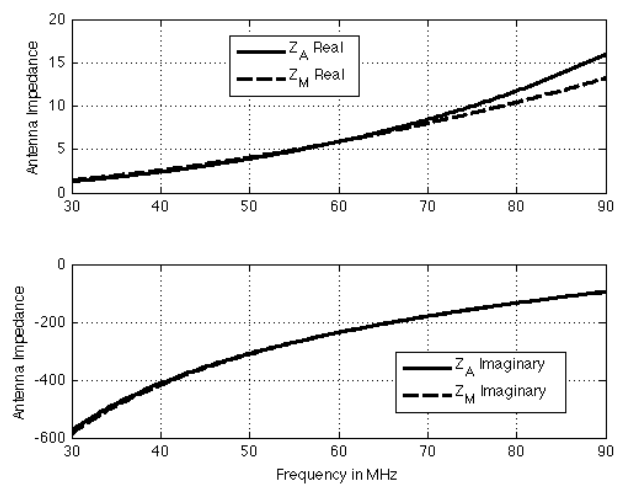

Fig. 1. Antenna model data and lumped element model calculations using MATLAB.

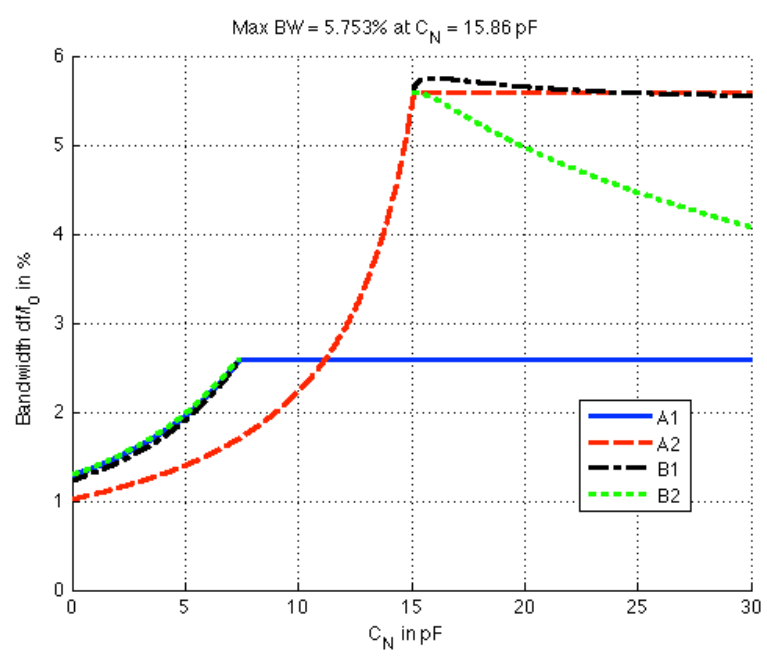

Fig. 2. MATLAB plot of bandwidth versus shunt negative capacitance values for different possible $L$-section matching network topologies.

time-domain MATLAB calculations of the node voltages and branch currents are shown in Figure 4. The conclusion is that this network is stable, as the currents and voltages approach zero asymptotically, for the specified parameters and initial conditions. These calculations have been verified in ADS.

$S$-domain analysis (omitted here for brevity) revealed that there will be a pole in the right half-plane for $\left(C_{M}+C_{N}+C_{A}\right)>0$. It will then be assumed that $C_{M}$ and $C_{N}$ are fixed, and the parameters that can change are those associated with the impedance of the antenna model. This would represent the antenna being altered, damaged, or in the vicinity of other objects, which would be a realistic concern. The underlying focus here is to determine what, if any, changes in antenna impedance may result in instability of the network that includes the antenna. So fixing the two capacitances at the values determined $\left(C_{M}=4.45 \mathrm{pF}\right.$ and $C_{N}=-15.86 \mathrm{pF}$ ), a pole arises in the right half-plane for $C_{A}>11.41 \mathrm{pF}$. To test this analysis, the value for $C_{A}$ has been changed to $12 \mathrm{pF}$. Results from MATLAB are shown in Figure 5, demonstrating an exponential growth in voltage and current for this case. 


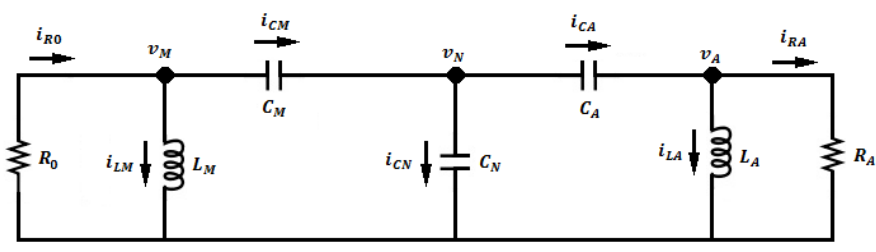

Fig. 3. Non-Foster matching network including lumped-element antenna model, ideal shunt negative capacitor, and passive matching network.

TABLE 1

ELEMENT VALUES FOR NON-FOSTER NETWORK

\begin{tabular}{|c|c|c|}
\hline Element & Description & Value \\
\hline $\boldsymbol{C}_{\boldsymbol{A}}$ & Antenna Model Capacitor & $8.54 \mathrm{pF}$ \\
\hline $\boldsymbol{L}_{\boldsymbol{A}}$ & Antenna Model Inductor & $200 \mathrm{nH}$ \\
\hline $\boldsymbol{R}_{\boldsymbol{A}}$ & Antenna Model Resistor & $955.8 \mathrm{Ohms}$ \\
\hline $\boldsymbol{C}_{\boldsymbol{N}}$ & Shunt Ideal Negative Capacitor & $-15.86 \mathrm{pF}$ \\
\hline $\boldsymbol{C}_{\boldsymbol{M}}$ & Series Matching Network Cap. & $4.45 \mathrm{pF}$ \\
\hline $\boldsymbol{L}_{\boldsymbol{M}}$ & Shunt Matching Network Ind. & $202.62 \mathrm{nH}$ \\
\hline $\boldsymbol{R}_{\boldsymbol{0}}$ & Trans. Line Impedance & $50 \mathrm{Ohms}$ \\
\hline
\end{tabular}

TABLE 2

DIFFERENTIAL EQUATIONS GOVERNING NONFOSTER NETWORK

\begin{tabular}{|c|}
$\frac{d v_{M}}{d t}=-\left[\frac{1}{R_{0} C_{N}}+\frac{1}{R_{0} C_{M}}\right] v_{M}-\frac{1}{R_{A} C_{N}} v_{A}-\left[\frac{1}{C_{N}}+\frac{1}{C_{M}}\right] i_{L M}-\frac{1}{C_{N}} i_{L A}$ \\
\hline$\frac{d v_{A}}{d t}=-\frac{1}{R_{0} C_{N}} v_{M}-\left[\frac{1}{R_{A} C_{N}}+\frac{1}{R_{0} C_{A}}\right] v_{A}-\frac{1}{C_{N}} i_{L M}-\left[\frac{1}{C_{N}}+\frac{1}{C_{A}}\right] i_{L A}$ \\
$\frac{d i_{L M}}{d t}=\frac{1}{L_{M}} v_{M}$ \\
$\frac{d i_{L A}}{d t}=\frac{1}{L_{A}} v_{A}$ \\
\hline
\end{tabular}

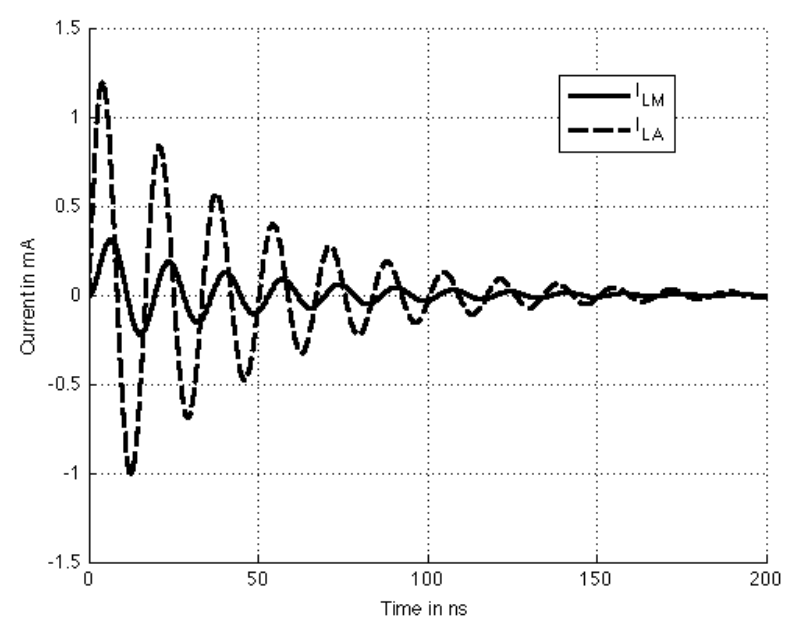

Fig. 4. MATLAB calculations based on differential equations of Table 2 of voltages and currents, $C_{A}=8.54 \mathrm{pF}$.

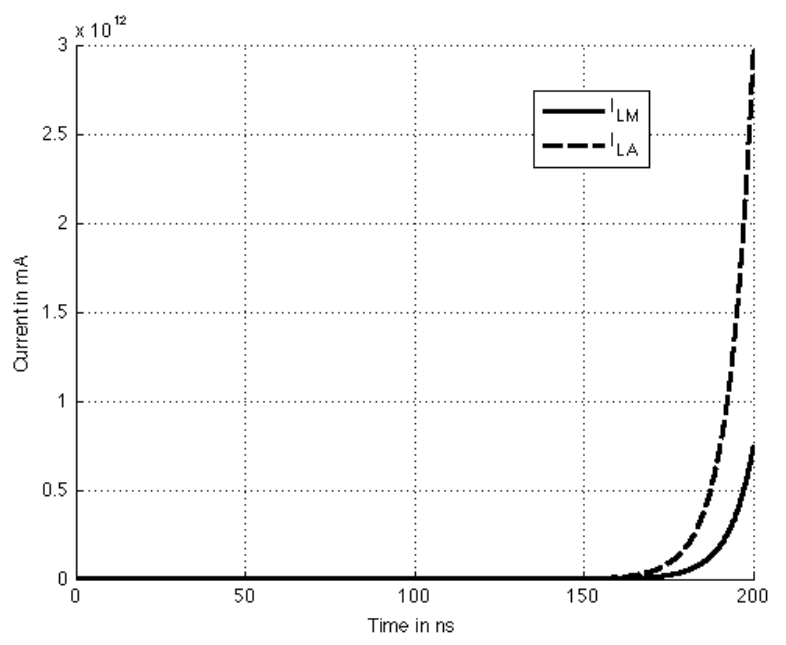

Fig. 5. MATLAB calculations based on differential equations of Table 2 of voltages and currents, $C_{A}=12 \mathrm{pF}$. 


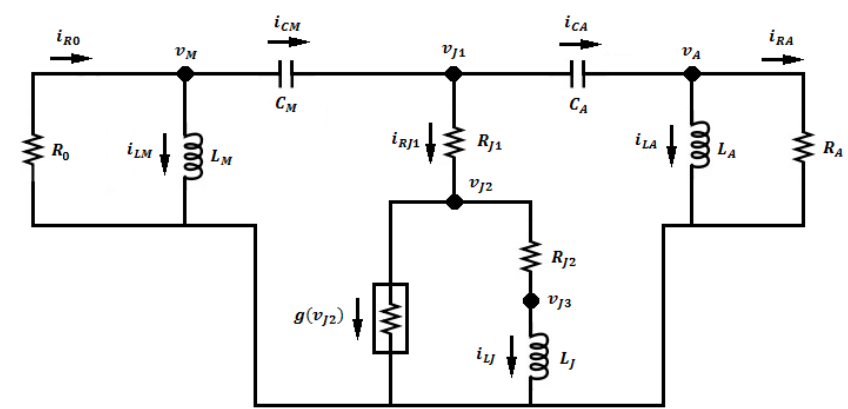

Fig. 6. Non-Foster network with nonlinearity.

TABLE 3

DIFFERENTIAL EQUATIONS GOVERNING NONFOSTER NETWORK WITH NONLINEARITY

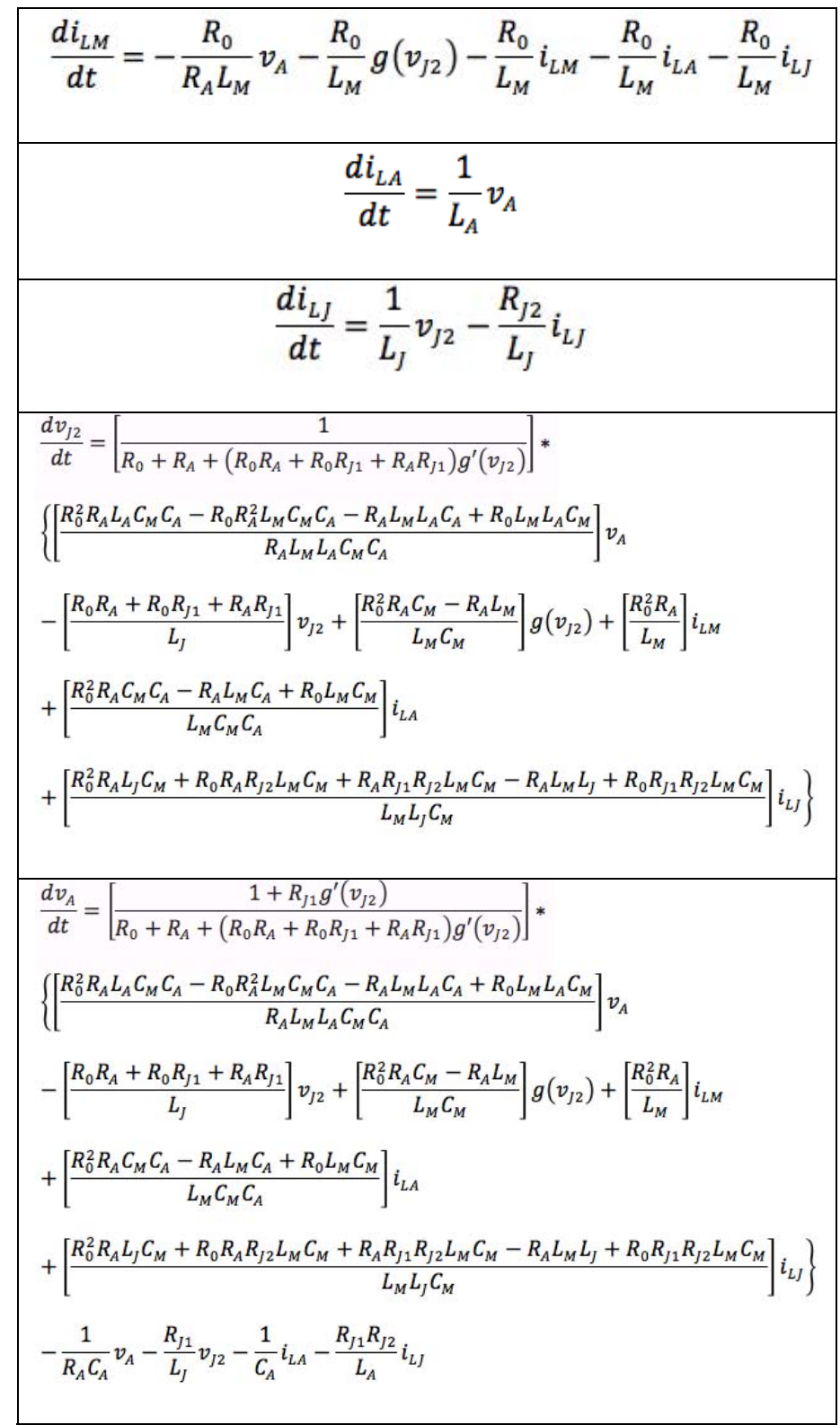

\section{NETWORK WITH NONLINEARITY}

Our ultimate goal is to develop a general, nonlineardynamics based paradigm to analyze the stability of nonFoster networks. Thus far, only an ideal negative capacitance has been considered. Here, the non-Foster network is changed such that an equivalent $J$-transform (using a negative resistor) is used instead of an ideal negative capacitor. Then the negative resistor is replaced with a nonlinear negative resistance. The resulting network is shown in Figure 6 . Nonlinear differential equations have been developed for this network, and are shown in Table 3. This set of nonlinear differential equations is currently being analyzed, and results will be presented at the conference.

\section{REFERENCES}

[1] R. M. Foster, "A Reactance Theorem," Bell System Technical Journal, Vol. 3, No. 2, pp. 259-267, April 1924.

[2] S. E. Sussman-Fort, "Non-Foster Impedance Matching of Electrically-Small Antennas," IEEE Transactions on Antennas and Propagation, Vol. 57, No. 8, pp. 2230 - 2241, August 2009.

[3] J. T. Aberle, "Two-Port Representation of an Antenna With Application to Non-Foster Matching Networks," IEEE Transactions on Antennas and Propagation, Vol. 56, No. 5, pp. 1218 - 1222, May 2008.

[4] R. L. Romack, Non-Foster Impedances as Active Broadband Matching Networks for Electrically Small Antennas, Master's thesis, Arizona State University, 2006.

[5] N. Dallman, General Stability Analysis of Networks Containing Negative Resistors, Master's thesis, Arizona State University, 2009.

[6] S. D. Stearns, "Incorrect Stability Criteria for Non-Foster Circuits," Proceedings of the 2012 IEEE Int. Symp. on Antennas and Prop. (APS / URSI), July 2012.

[7] T. Matsumoto, L. O. Chua, M. Komuro, "The Double Scroll," IEEE Transactions on Circuits and Systems, Vol. cas-32, No. 8, August 1985.

[8] A. Kandangath, S. Krishnamoorthy, Y.-C. Lai, J. A. Gaudet, "Inducing Chaos in Electronic Circuits by Resonant Perturbations," IEEE Transactions on Circuits and Systems - I: Regular Papers, Vol. 54, No. 5, May 2007.

[9] L.J. Chu, Physical Limitations of Omnidirectional Antennas, Tech. Rept. No. 64, Research Laboratory of Electronics, MIT, May 1, 1948. Published in J. Applied Physics, vol. 19, no. 12, pp. 1163-1175, Dec. 1948.

[10] D. M. Pozar, Microwave Engineering, Third Edition, New York, Wiley, 2005. 